\title{
Les Chemins de Saint-Gilles au Moyen Âge
}

\section{Pierre-Gilles Girault}

\section{(2) OpenEdition \\ Journals}

Édition électronique

URL : https://journals.openedition.org/geohist/447

DOI : 10.4000/geohist.447

ISSN : 2264-2617

Éditeur

Association française de la Revue de géographie historique

\section{Référence électronique}

Pierre-Gilles Girault, "Les Chemins de Saint-Gilles au Moyen Âge », Revue de géographie historique [En ligne], 16 | 2020, mis en ligne le 20 mai 2020, consulté le 12 juin 2021. URL : http://

journals.openedition.org/geohist/447 ; DOI : https://doi.org/10.4000/geohist.447

Ce document a été généré automatiquement le 12 juin 2021.

\section{cc) (1) $\odot$}

Ce(tte) œuvre est mise à disposition selon les termes de la Licence Creative Commons Attribution -

Pas d'Utilisation Commerciale - Pas de Modification 4.0 International. 


\title{
Les Chemins de Saint-Gilles au Moyen Âge
}

\author{
Pierre-Gilles Girault
}

1 Tout pèlerinage s'inscrit dans l'espace au moins à trois titres : par la localisation - et l'aménagement - du sanctuaire qui en est le but; par l'origine géographique des pèlerins qui s'y rendent; enfin par les routes ou itinéraires que les seconds empruntent pour se rendre vers les premiers.

2 Il peut paraître singulier de classer parmi «les grands pèlerinages » le sanctuaire, aujourd'hui assez méconnu, de Saint-Gilles (Gard), aux confins de la Provence et du Languedoc, souvent regardé aujourd'hui comme une simple étape vers Compostelle. Toutefois, si le fameux Guide du pèlerin de Saint-Jacques fait de Saint-Gilles une étape et le point de départ d'une route vers Compostelle, cette allégation paraît bien relever de la dimension mythique du chemin. En effet, Gérard Jugnot a naguère signalé que, pour les itinéraires conservés ou pour les pèlerins de Saint-Jacques dont le trajet est connu, " aucun ne prend son départ à Arles » et que, ajoutait-il, « aucun ne passe par SaintGilles-du-Gard» (Jugnot, 1982, p. 65). Or Saint-Gilles a bien été d'abord le but d'un pèlerinage très couru au Moyen Âge, notamment au XII ${ }^{\mathrm{e}}$ siècle, comme l'attestent plusieurs sources (Sigal, 1993 ; Girault, 2012). Mon père Marcel Girault a consacré sa vie à le faire revivre depuis 1965 sous le nom justement évocateur des « chemins de SaintGilles » et à en reconnaître les itinéraires sur le terrain, notamment le fameux chemin de Regordane (Girault 1980, 1986, 1990).

3 En décrivant Saint-Gilles comme étape, voire comme point de départ vers SaintJacques, ou par le nom de via egidiana, "route de Saint-Gilles », qu'il applique à son itinéraire méridional, le Guide du pèlerin nous paraît dicter notre questionnement : des chemins de Saint-Gilles ont-ils existé ? Quels rapports entretiennent-ils avec ceux de Saint-Jacques? Pour l'appréhender, il nous faut recourir à des sources diverses : outre le Guide du pèlerin lui-même, le Liber miraculorum sancti Egidii (Girault, 2007), les fragments d'itinéraires glanés à travers chroniques, journaux ou livres de raison, mais aussi sources littéraires, notamment épiques, dont nous avons souligné ailleurs à la 
suite de Joseph Bédier (1908-1912), la richesse pour l'étude des pèlerinages (Girault, 2001).

\section{Les itinéraires du Guide du pèlerin de Saint-Jacques}

\section{A. La via Egidiana}

Notre connaissance des itinéraires de pèlerinage médiévaux repose pour beaucoup sur le Guide du pèlerin, titre sous lequel on a pris l'habitude de désigner le livre V du Liber sancti Jacobi conservé dans le Codex Calixtinus de Compostelle (Vielliard, 1950 ; Herbers, Santos Noia, 1998; Gicquel, 2003). Ce texte fameux, tout entier tendu vers SaintJacques, n'en mentionne pas moins Saint-Gilles à plusieurs reprises. Le célèbre prologue décrivant les quatre voies, qui selon l'auteur, conduisent à Compostelle, est trop connu pour que j'aie besoin de le rappeler ici ; soulignons seulement que c'est la première des routes citées que l'auteur dit passer par Saint-Gilles, puis Montpellier, Toulouse et le Somport.

C'est justement sur la via egidiana ou tolosana, ce " grand axe routier est-ouest du Midi de la France » (Bautier, 1963), que la ville d'Arles apparaît dans les sources littéraires comme un lieu de passage de pèlerins tant vers Saint-Jacques que vers Saint-Gilles (Bédier, 1908-1912, t. I, p. 354-360 et 364-424). Rendant compte des chevaliers chrétiens qui ont trouvé la mort en affrontant les Sarrasins, l'auteur de la chanson de geste de la Chevalerie Vivien allègue le témoignage des pèlerins de Compostelle qui ont vu aux Aliscamps d'Arles la terre rouge de leur sang et leurs innombrables tombeaux : Encore le voient li pelerin assez, / Qui a Saint Jacque ont le chemin torné (éd. Terracher, 1909, vv. 1788-1789). Une variante de ce vers rencontrée dans les manuscrits de Londres et de Paris en appelle quant à elle aux pèlerins de Saint-Gilles, li pelerins... Qui a Saint Gille ont le chemin torné (Bédier, 1908, t. I, p. 402, n. 2). Ce n'est pas là licence poétique car une terre, bien réelle celle-ci, située entre Arles et Saint-Gilles est dite en 1192 jungitur camino quo itur ad S. Egidium (Gilles-Guibert, 1962, p. 6).

D'autres poèmes soulignent le rôle de Saint-Gilles comme étape vers Rome, souvent sans préciser si le voyage est accompli par voie de terre ou par voie de mer. La chanson Elie de Saint-Gilles proclame que passent par la ville provençale des pèlerins qui s'en vont à Rome prier l'Apôtre: Tu peus encontrer pelerin ne paumier / Qui en aut a Saint Gille, por l'apostle proier (éd. Raynaud, 1879, v. 1339-1340). Dans le poème Ami et Amile, quand ses serviteurs, Garins et Haymmes, conduisent le héros chez son parrain, à Rome, ils annoncent qu'ils feront étape à Saint-Gilles : Nos voz menrons desci jusqu'a Saint Gile (éd. Dembowski, 1969, v. 2418-2420). Dans l'autre sens, plusieurs poèmes attestent le passage de pèlerins réels ou supposés qui font étape à Saint-Gilles à leur retour de Rome. Dans Aye d'Avignon, le traître Ganor prétend qu'il revient de Rome en passant par Saint-Gilles de Provence (Puis sui venus de Ronme par Saint Gile en Prouvence) pour continuer sa route vers Le Puy et même Tours (éd. Borg, 1967, v. 2408).

Dans le roman L'Escoufle écrit vers 1200 par Jean Renart, le héros, Guillaume de Montivilliers, quitte Rome et se met en chemin jusqu'à Saint-Gilles : Dusqu'a S. Gilles as grans jornees. / Tant a levés as jornees / Ke il vint a S. Gille droit (éd. Sweetser, 1974, v. $6489,6490,6492)$. Le cas le plus piquant est celui du roi païen Cornumaran, dans la chanson de Godefroid de Bouillon, qui, déguisé en pèlerin, parvient à Rome, d'où il projette de se rendre à Saint-Gilles afin d'y rencontrer le comte Raimond de Toulouse, 
en traversant les Alpes par le col de Montjoie, c'est-à-dire au Grand-Saint-Bernard : Cele nuit ont andoi à Rome sejorné. / El demain s'en revont, si ont Mon-Jeu passé. / Quant sont outre les mons, s'a li rois devisé / Qu'il ira a Saint Gille (éd. Hippeau, t. II, Paris, 1877, v. 2547-2550).

\section{B. Route de Toulouse ou de Saint-Gilles?}

8 Mais revenons à l'itinéraire du Guide du pèlerin. Comme le remarque Gérard Jugnot, la route décrite dans le Guide « est qualifiée de via tolosana ou encore de via egidiana : où le chemin de Saint-Jacques des uns est, de toute évidence, le chemin de Saint-Gilles des autres » (Jugnot, 1982, p. 61)... Y aurait-il donc des attestations de pèlerins venus par cette route de Toulouse à Saint-Gilles?

9 Le Livre des miracles de saint Gilles montre peu de pèlerins d'origine toulousaine. Il mentionne toutefois un moine sauvé de la noyade alors qu'il « essayait de passer à gué la Garonne dont les eaux arrosent Toulouse » et qui vient rendre grâce à Saint-Gilles (Ibid., n²9, p. 200-201). Que la route de Toulouse soit également empruntée en direction de Saint-Gilles ressort encore de la chanson de geste des Enfances Guillaume. Dans le poème, le comte Aymeri de Narbonne, passe Toulouse et rallie Saint-Gilles pour y placer ses fils sous la protection divine : Cuens Ainmeris, quant Tolouse ait pacey, / Droit vers Saint Gille ait son chamin torney: / A cort an moinne ces anfans por oreir. / Deus les gairisent, li rois de maïstei (éd. Henry, 1935, v. 315-324)!

Outre Arles et Toulouse, il est deux autres villes citées dans le Guide où le passage de pèlerins de Saint-Gilles est avéré, Montpellier et Saint-Guilhem (Macaire, 2000). Elles apparaissent aussi comme des étapes communes à une autre route, celle de Notre-Dame de Rocamadour. C'est l'itinéraire suivi en 1169 par l'évêque de Londres Gilbert pour se rendre à Rome via Milan : "Il prit la route par un chemin très long et très difficile, passant par Rocamadour, par Saint-Guilhem, par Montpellier, par Saint-Gilles et après avoir traversé les Alpes, il parvint à Saint-Ambroise » (Albe, 1907, p. 53 ; Girault, 1990, p. 193-247).

11 L'étape de Montpellier, citée dans le premier chapitre du Guide du pèlerin, paraît en outre attestée par un passage du sermon Veneranda dies. L'auteur, soi-disant le pape Calixte II, y relate une rixe peut-être observée lors de son séjour à Saint-Gilles en 1119, qui opposa dans la crypte de l'abbatiale des pèlerins français et gascons et observe que l'un d'eux, «blessé à la tête, s'enfuit jusqu'à Châteauneuf par la route de Périgueux ( $a d$ Castellum Novum via Petragoricensi) et y mourut ». Nous proposons de traduire Castellum Novum non par Châteauneuf, mais plutôt par Castelnau, toponyme qui désigne probablement Castelnau-le-Lez, près de Montpellier (Liber sancti Jacobi, 1998, p. 94. Gicquel, 2003, p. 365). Le passage des pèlerins y est en effet documenté par la mention d'une "route publique appelée chemin des pèlerins" (publica via que peregrinorum caminus vocatur) appliquée au pont de Castelnau dans un traité de 1132 (Bautier, 1963, p. 289 ; Girault, 1990, p. 236). L'itinéraire suivi par le blessé vers Périgueux correspondrait ainsi à celui suivi dans l'autre sens par l'évêque Gilbert cinquante ans plus tard.

D'autres toponymes attestent le passage par Montpellier de pèlerins en route tant vers Rome et Saint-Gilles que vers Saint-Jacques: le nom de cami romieu donné à la voie Domitienne puis au chemin parallèle à l'ancienne voie, dit caminus romeus novus; la mention camino romeo appliquée en 1344 au tronçon de route compris au nord de la ville entre Vendargues et Saint-Brès; la mention via qua itur de Pruneto ad caminum 
sancti Jacobi qui, vers 1200, désigne à Saint-Martin-de-Prunet le chemin rejoignant la grande route de Montpellier à Nîmes; enfin l'expression via publica sancti Jacobi appliquée en 1246 au «chemin roumieu neuf » déjà évoqué au sud, à Gigean, en direction de Béziers (Gilles-Guibert, 1962, p. 32-33).

Une autre étape vers Saint-Jacques indiquée par le Guide du pèlerin, celle de SaintGuilhem-le-Désert, apparaît aussi dans les sources littéraires. La rédaction II du Moniage Guillaume rapporte que Guillaume d'Orange décide de bâtir à l'intention des pèlerins un pont de pierre sur l'Hérault, le célèbre Pont du Diable : La se voldront pelerin adrechier, / Quant il iront a Saint Gille proier; Par la iront Rochemadoul poier, A Nostre Dame qui en la roche siet (éd. Cloetta, 1906-1913, t. II, v. 6560-6563). Cet extrait montre bien que le pont de Saint-Guilhem constitue le point de passage d'un itinéraire qui peut être emprunté par les pèlerins tant vers Saint-Gilles que vers Rocamadour.

\section{Le Puy à la croisée des chemins}

Un autre point de départ de l'un des itinéraires décrits par le Guide du pèlerin rencontre aujourd'hui la faveur de nombreux marcheurs. Il s'agit de la ville du Puy-en Velay, qui fut le point de départ de l'évêque Godescalc, premier pèlerin français attesté à Compostelle en 951 (Péricard-Méa, 2000, p. 188). Toutefois le Puy n'est pas seulement un point de départ vers la Galice : c'est aussi et surtout un éminent pèlerinage à la Vierge (Moulinet, Maes, Vincent, 2009) ; c'est enfin une étape vers d'autres sanctuaires que Compostelle et en particulier vers Saint-Gilles.

En effet, au cœur de la ville du Puy, dans l'angle sud-ouest de la place du Plot, où se rassemblaient les pèlerins sur le départ, s'ouvrent encore deux rues perpendiculaires : la rue Saint-Jacques et la rue Saint-Gilles. Si la rue Saint-Jacques marque le départ du chemin de Compostelle, on sait moins que la rue Saint-Gilles se prolonge elle aussi audelà des limites de la ville et conduit à l'abbatiale de Saint-Gilles du Gard. Cette rue Saint-Gilles du Puy est citée depuis le XIII siècle, carriera Sancti Egidii (1286) Caminus S. Aegidii en 1323 et 1335, rue sainct Gire en 1544, rue Sainct-Gilles en 1585 et depuis le XVIII ${ }^{e}$ siècle (Chassaing, 1869-1874, t. I, p. 271 et t. II, p. 609). Elle aboutissait au rempart dans lequel s'ouvrait la porte Saint-Gilles, la porte sainct Gile citée depuis le XIV ${ }^{\mathrm{e}}$ siècle. $\mathrm{Au} \mathrm{XVI}{ }^{e}$, Le livre de Podio d'Etienne Médicis, confirme que cette porte était bien celle par laquelle sortaient les pèlerins en route vers l'abbaye provençale : "La sixième porte s'appelle de sainct Gilles, pour ce que de ceste porte on yst de ladicte ville du Puy pour aller en l'antique ville de Sainct Giles là-bas en Languedoc » (Chassaing, 1869-1874, t. II, p. 273).

16 S'il n'existe pas pour Saint-Gilles d'équivalent au Guide du pèlerin de Saint-Jacques, on dispose pour le Moyen Âge de plusieurs itinéraires plus ou moins détaillés qui attestent du passage au Puy des pèlerins de Saint-Gilles du $\mathrm{XI}^{\mathrm{e}}$ au XIII ${ }^{\mathrm{e}}$ siècle.

\section{Les sources des Chemins de Saint-Gilles}

\section{A. Le pèlerinage de Robert le Pieux}

Si on excepte l'abbé de Figeac, Adacius, pèlerin de Rome, qui serait mort d'épuisement en 988 en arrivant à Saint-Gilles (Girault, 1990, p. 196), ou le comte d'Urgel Ermengaud $\mathrm{I}^{\mathrm{er}}$, qui lègue en 1008, des pièces de son jeu d'échecs à l'église de Saint-Gilles 
(Pastoureau, 2004, p. 269 et 276), le plus ancien pèlerin de Saint-Gilles attesté par une source contemporaine n'est autre que le roi de France Robert le Pieux (972-1031).

Le récit de ce voyage est fourni dans la biographie de Robert le Pieux du moine Helgaud de Fleury composée peu après la mort du roi. Vers la fin de son récit, l'auteur annonce : "Cet excellent roi, qui voulait mourir au siècle, et ne vivre que pour Jésus-Christ [...], se mit donc en route un jour de carême pour aller visiter les saints qui lui étaient unis dans le service de Dieu ; il les pria [...] et employa à cela la force de son corps et de son esprit afin de vaincre, par l'aide de Dieu. Il arriva à Bourges, visita le saint protomartyr Etienne, ainsi que saint Maïeul, grand par sa vertu ; sainte Marie et l'illustre et grand Julien ; il gagna la très pieuse Vierge des vierges Marie ; saint Gilles grand confesseur ; le fameux Saturnin, le courageux Vincent, Antonin, digne du martyre, saint Géraud, brave soldat du Seigneur; et revint sain et sauf au glorieux Etienne auprès duquel il passa joyeusement le dimanche des Rameaux. De là il se rendit à Orléans, pour y recevoir, le jour de Pâques, l'auteur de notre salut » (Guizot, 1824, p. 406-407 ; Bautier, Labory, 1965, p. 126).

À la suite des Historiens de la France et de Robert-Henri Bautier, on admet en général que ce pèlerinage se situe peu avant la mort du roi et s'est déroulé durant le carême 1030 voire 1031. Robert le Pieux aurait ainsi, pendant la mauvaise saison, visité les plus grands sanctuaires de son temps : Saint-Étienne de Bourges, Saint-Mayeul de Souvigny, Notre-Dame de Clermont, Saint-Julien de Brioude, Notre-Dame du Puy et Saint-Gilles, avant de rentrer par Saint-Vincent de Castres, Saint-Sernin de Toulouse, Saint-Antonin de Noble-Val, Sainte-Foy de Conques et Saint-Géraud d'Aurillac, sans doute en suivant l'itinéraire pèlerin d'alors.

Dans sa thèse sur l'Auvergne et le Velay du Haut Moyen Âge, Christian Lauranson-Rosaz (1987) a proposé une interprétation différente de ce passage. Il propose d'étendre à deux années ce voyage commencé un jour de carême et terminé à Pâques, alors qu'on le pensait jusque là accompli en 40 jours. Il suggère en outre de le dater des années 1019-1020, plutôt que de la fin du règne. La portée du voyage s'en trouve modifiée : au lieu du pèlerinage religieux d'un roi âgé soucieux de son salut évoqué par Helgaud, il s'agirait d'une tournée avant tout politique dans le sud du royaume visant à pacifier l'Auvergne et à affirmer l'autorité du roi. Ces changements de perspective ont été acceptés par la critique et notamment par le dernier biographe du roi (Theis, 1999). En vérité, si les arguments sont recevables, aucun ne paraît décisif. Seule une charte nîmoise de juillet 1019 qui fait mention de la générosité du roi pourrait faire allusion au récent passage de Robert et constitue en définitive l'unique argument en faveur d'une datation du voyage en 1019.

21 Quoi qu'il en soit, la Vie de Robert le Pieux brosse à grands traits un itinéraire nord-sud d'Orléans à Saint-Gilles via Bourges, Souvigny, Clermont, Brioude et Le Puy que d'autres sources au siècle suivant nous confirment être la principale voie d'accès au sanctuaire provençal.

\section{B. Le Charroi de Nîmes}

La première partie de l'itinéraire royal suit en effet le « chemin de saint Gilles » tel qu'il est décrit vers 1130-1140 dans la chanson de geste du Charroi de Nîmes, qui est l'une des plus anciennes épopées françaises (éd. Perrier, 1931; éd. et trad. Lachet, 1999). Le poème relate l'expédition militaire conduite par Guillaume d'Orange pour délivrer 
Nîmes de l'occupation sarrasine. Sans en être le but, Saint-Gilles et son pèlerinage dominent tout le poème et sont sa raison d'être. En effet, si Guillaume veut reprendre Nîmes aux Sarrasins, c'est qu'il a fait vœu de délivrer la ville à l'occasion d'un pèlerinage à Saint-Gilles, Quant $a$ s. Gile alai pour Dieu proier (ms. A2, v. 549). C'est ce vœu qui fournit l'argument du récit, c'est en pèlerin que le poète a conçu son héros et c'est l'itinéraire des pèlerins que celui-ci emprunte avec son armée. Le jongleur achève d'ailleurs son poème sur ce rappel: Ceste canchon que vous m'oes dire, / Est en la voie de monseignor saint Gille (C, v. 1103-1104). La précision de l'itinéraire du Charroi a été reconnue depuis longtemps : Joseph Bédier (1908, t. I, p. 369-394) l'a analysée et Marcel Girault (1982) en a précisé la toponymie.

L'armée que Guillaume a constituée sort de Paris par Petit-Pont, puis traverse le Berry et l'Auvergne, Ont trespassé et Berri et Auvergne (A, v. 832). Elle contourne par l'est Montferrand et Clermont qu'elle laisse à droite, Clermont lesserent et Montferent a destre (A, v. 833). Plus loin, Guillaume ordonne à ses barons d'emprunter le chemin de Saint-Gilles vers Brioude et Notre-Dame du Puy : Tot le chemin de saint Gilles tenés, / Tot droit à Bride la vous aceminés, / Tot droit au Pui ou est la mere Dé (éd. Perrier, ms. C, v. 858-860 ; Girault, 1982, p. 1106, 1115).

Chevauchant à travers bois et forêts, Guillaume et ses hommes traversent ensuite le pays de Regordane, Par Ricordane outre s'en trespasserent (A, v. 840), territoire qui a donné son nom au chemin qui le traversait, improprement appelé Voie Regordane depuis quelques décennies, malgré les formes anciennes attestées: Itinere Regordane, le grand camy de Regordane, Chemin de Regordane.

La troupe poursuit sa route jusqu'à Alès, Dusc'a l'Areste ne se sont aresté (C, v. 883). Puis elle passe sous Vézenobres, Par Ville Noble en sont outre passé(D, v. 1011), franchit le Gardon à gué au moyen d'une chaussée submersible, Sur la chaucie passe Gardone au gué (A-B, v. 1032), ou bien à gué à proximité de La Calmette, $A$ la Charmaite passent Guaidons au gués (D, v. 1012). Enfin, Guillaume et ses hommes passent à proximité des carrières romaines de Barutel, qu'il appelle Bremarle, dont furent extraites les pierres des arènes de Nîmes, Dont les granz tors de Nimes furent faites (D, vv. 1050-1054).

L'auteur du Charroi connaît aussi Monclar, Montcler, piton rocheux dominant les vallées du Luech et de l'Homol, à l'extrémité orientale du Mont Lozère (B, v. 890). Il cite aussi Laval, Laval desus Cler, dont le sanctuaire marial proche de La Grand-Combe est connu depuis le IXe siècle (A, v. 890). Un paysan rencontré sur la route, lui aussi, Vient de Saint Gile (A, v. 876). Interrogé sur ce qu'il a vu à Nîmes, il rapporte les difficultés que connaissent les pèlerins contraints de s'acquitter d'un lourd péage (C, vv. 1107-1115). Enfin, le terme du voyage, la cité de Nîmes, ne peut être autrement situé que par rapport à Saint-Gilles: Ceste cité dont je vos chants, de Nymes, Est en la terre de mon seignor saint Gile (A, v. 1093).

\section{Autres attestations littéraires}

27 L'appellation de "chemin de saint Gilles » n'est pas propre au Charroi, mais attestée par d'autres sources. Dès 1108, le chroniqueur de l'abbaye de Saint-Pierre-le-Vif de Sens, qui séjourne dans le prieuré d'Orcet, situé au sud de Clermont sur la route de Brioude, le localise sur le chemin public qui va à Saint-Gilles : inpublica via qua ad Sanctum Egidium itur (Girault, 1980, t. I, p. 51 ; id., 1990, p. 93). De même, au sud du Puy 
cette fois, à Vals, la « route de Saint-Gilles » est citée en confront d'un champ dans un acte du XII ${ }^{\mathrm{e}}$ siècle (Girault, 1980, t. I, p. 53).

Quant à l'itinéraire du Charroi de Nîmes, il apparaît par bribes dans d'autres romans et chansons. Dans le roman de Guillaume d'Angleterre, le pieux roi Guillaume qui envoie ses deux fils en pèlerinage mentionne Le Puy et Saint-Gilles, S'iront au Pui et a Saint Gille (éd. Wilmotte, 1927, v. 2016). D'autres poèmes suggèrent le même itinéraire dans le sens sud-nord. Dans la chanson Aye d'Avignon, le faux pèlerin Ganor, situe Saint-Gilles sur un itinéraire qui le conduit de Rome au Puy et de là vers Tours : Puis sui venus de Romme par Saint Gile en Prouvence. / Au Pui Sainte Marie me convient a descendre, / A Saint Martin a Tours voudrai mon chemin prendre (v.2408-2410).

Mais, on le voit, l'itinéraire est désormais réduit à ses principales étapes, voire à la seule mention des régions traversées. Ainsi dans le poème Aiol, le héros, qui revient d'Espagne où il a enlevé sa femme Mirabel, accoste à Saint-Gilles où il accomplit ses dévotions et remonte à travers la Provence, où il ne reste guère, puis traverse l'Auvergne et le Berry pour atteindre Orléans : Et vinrent a Saint Gille al cors saint honoré. / Quant ont fait lor priere, si s'en sont retorné, / Et monterent es seles des mulés sejornés, / Et trespassent Provence, n'i ont gaires esté; / Il trespassent Alverne et Beri par delés, / Et viennent a Orliens la mirable chité (éd. Normand et Raynaud, 1877, v. 10457-10464).

Dans ces « voyages éclairs » brossés en quelques mots, une autre étape souvent citée est celle de Brioude. Le passage des pèlerins en route vers Saint-Gilles y est attesté par d'autres poèmes. Au sujet des armes que de nombreux héros épiques y auraient déposées, des chansons de geste invoquent le témoignage des pèlerins de Saint-Gilles qui y font étape. Ainsi, le Moniage Guillaume rapporte qu'Encore le voient et li fol et li sage / Tot chil qui vont a Saint Gille en voiage (éd. Cloetta, I, v. 94-95), tandis que l'auteur de La prise d'Orange renchérit : Icil le sevent qui en vont a Saint Gille / Qui les enseignes on ont veü a Bride (Girault, 1990, p. 93 et 136-137).

\section{L'itinéraire de l'archevêque de Rouen Eudes Rigaud}

31 C'est du XIII ${ }^{e}$ siècle que date le troisième itinéraire fondamental, celui suivi par l'archevêque de Rouen Eudes Rigaud en 1260 (Andrieu-Guitrancourt, 1938 ; Davis, 2006). L'itinéraire suivi par le prélat franciscain réformateur a été publié dès le XIX ${ }^{\mathrm{e}}$ siècle, ses étapes précisées par son éditeur et plusieurs travaux ultérieurs (Bonnin, éd., 1852; Darlington, 1940 ; Brown, O'Sullivan, 1964).

Parti de Rouen le 5 avril 1260, Eudes Rigaud traverse les actuels départements de l'Eure et de l'Eure-et-Loir. À Dreux le 9 avril, il séjourne deux jours à Chartres, puis à Janville. Evitant Orléans, il rejoint la Loire à Jargeau, longe le fleuve jusqu'à Gien, fait étape au manoir de l'évêque d'Auxerre à Villechaud le 15, le lendemain à Urzy, dans la demeure de l'évêque de Nevers (Nièvre), puis à Saint-Pierre-le-Moûtier.

Le 19, il est dans l'Allier à Moulins, puis à Saint-Pourçain-sur-Sioule, d'où il atteint le Puy-de-Dôme à Aigueperse. Gagnant ensuite Montferrand et Clermont (au manoir épiscopal), il rejoint là le traditionnel « chemin de Saint-Gilles » qui le conduit à Issoire, puis en Haute-Loire à Brioude et au Puy-en-Velay, en Lozère à Luc puis à travers les Cévennes dans l'actuel département du Gard à Génolhac, Alès, Nîmes et enfin SaintGilles. Le 2 mai, l'archevêque note dans son registre : « Nous visitâmes le sanctuaire de Saint-Gilles, et nous célébrâmes, par la grâce de Dieu, la messe sur l'autel qui jouxte le 
tombeau où repose le corps du saint, puis nous mangeâmes avec le vénérable père archevêque de Narbonne, et nous passâmes la nuit dans l'abbaye. "

Le lendemain, Eudes Rigaud va visiter le sanctuaire de Notre-Dame de Vauvert où il célèbre la messe et revient passer la nuit à Nîmes. Changeant d'itinéraire au retour, il suit la vallée du Rhône pour rejoindre l'Ile-de-France à travers les monts du Morvan. De Nîmes il gagne Bagnols-sur-Cèze, probablement en passant par Remoulins où est cité en 1244 un camino roumieu qui révèle le passage de pèlerins (Gilles-Guibert, 1962, p. 32). Il rejoint ensuite le Rhône, atteint la Drôme à Montélimar puis Valence et, longeant toujours le fleuve, atteint Roussillon (Isère) puis Lyon (Rhône) où il partage le repas des Frères mineurs le 9 mai. Il oblique alors au nord-ouest, fait étape à L'Arbresle, à Charlieu (Loire) et atteint la Haute-Saône à La Motte-Saint-Jean, puis traverse la Nièvre en trois jours par Montécot (sur la commune de Sémelay), Moulins-Engilbert où il célèbre la messe de l'Ascension (14 mai) et accomplit ses dévotions à Saint-Léonard de Corbigny.

Il séjourne cinq jours dans l'Yonne, à Asquins, au-dessous de Vézelay, à Régny ou Reigny, manoir de l'évêque d'Auxerre, puis à Villeneuve-sur-Yonne où il rencontre le roi qu'il accompagne les jours suivants à Sens puis à Montereau-Fault-Yonne et Melun (Seine-et-Marne). Arrivé à Corbeil-Essonnes (Essonne), il célèbre la messe de la Pentecôte dans la chapelle royale. Passant par Villeneuve-Saint-Georges, il atteint Paris le 18 mai. De là, il regagne la Normandie en passant par Meulan (Yvelines), l'abbaye de Saulseuses, sur la commune de Bois-Jérôme-Saint-Ouen (Eure), Fresne-le-Plan (SeineMaritime) après un détour par Les Andelys. Il est de retour à Rouen le 31 mai.

L'itinéraire d'Eudes Rigaud, tracé au plus court, valide étape par étape le «chemin de Saint-Gilles " à partir de Clermont, mais souligne aussi l'existence d'autres voies d'accès : celle du Rhône et, pour les relier, des voies bourguignonnes : l'une à travers le Nivernais, au lieu de la route passant par Orléans et Bourges rencontrée précédemment ; la seconde, empruntée au retour, traversant la Bourgogne tout entière du sud au nord. L'étape de Vézelay, point de départ de la troisième route citée par le Guide du pèlerin de saint Jacques, constituait un autre carrefour où se rencontraient pèlerins de Compostelle et de Saint-Gilles, d'autant plus significatif que l'église du village d'Asquins situé au pied de la colline était justement consacrée à l'apôtre de Galice.

La route bourguignonne suivie par Rigaud à son retour est sans doute celle que suivaient les pèlerins de Saint-Gilles venus du Nord et de l'Est de la France. C'est aussi la route que choisit le héros épique Bernier, dans la chanson de Raoul de Cambrai, lorsqu'il part à la recherche de son fils, Julien, que les Sarrasins ont enlevé. Parti de Cambrai, Bernier et son compagnon traversent la France (c'est-à-dire l'Ile-de-France) et la Brie ; à Sens, ils entrent en Bourgogne et de là gagnent rapidement Saint-Gilles:France trespassent et Brie par dalés, / Par endroit Sens sont en Borgoingne entrés. / De lors jornées ne vous sai aconter. / Dusqu'a Saint Gile ne se sont arestés (éd. Meyer et Longnon, 1882, v. 7657-7660 ; éd. et trad. Kay et Kibler, 1996, v. 7474-7477).

Leur itinéraire n'est pas autrement précisé mais pourrait bien emprunter ce «Chemin de Saint-Gilles » repéré par Marcel Girault sur une dizaine de kilomètres à travers plusieurs communes de l'Yonne (Béon, Chamvres, Champvallon, Senan, Volgré, Villierssur-Tholon et Aillant), situées dans le canton d'Aillant-sur-Tholon (à l'ouest d'Auxerre) et dont Marcel Girault retrouve un court tronçon, $30 \mathrm{~km}$ plus au sud, sur le territoire des communes de Fontenoy et de Saints. 
bas, le Chemin de Saint-Gilles bourguignon pouvait également passer par BussyAlbieux (Loire), comme l'indique en 1046 une lettre de l'archevêque de Lyon confirmant la donation faite par un certain Aschirius à l'abbaye de Savigny (Rhône). Celui-ci cède au monastère l'église située à Bussy, ainsi que les offrandes faites aux patrons de l'église, Notre-Dame et saint Galmier, «par les pèlerins et romieux en route vers les sanctuaires des saints, tant à Sainte-Marie qu'à Saint-Pierre, non moins qu'à SaintJacques et à Saint-Gilles ", Donamus etiam oblationes quas attulerint homines peregrini et romei, et aliorum extraneorum pergentium ad aliquem sanctum, tam ad beatam Mariam, quam ad beatum Petrum, necnon ad sanctum Jacobum et ad sanctum Aegidium (éd. Bernard, 1853, t. I, $\left.\mathrm{n}^{\circ} 731, \mathrm{p} .378\right)$. On ignore si le premier sanctuaire mentionné désigne Sainte-Marie Majeure ou, plus vraisemblablement, Notre-Dame du Puy, comme l'ont proposé Maurice Valla (1964) et Marcel Girault (1990, p. 339-340). Cet acte montre que dès le milieu du XI ${ }^{\mathrm{e}}$ siècle, des pèlerins traversant le Forez se dirigent, via Le Puy, tant vers Saint-Jacques que vers Saint-Gilles.

\section{E. Saint Louis pèlerin}

L'itinéraire très détaillé fourni par Eudes Rigaud peut être recoupé dans ses grandes lignes par ceux d'un autre pèlerin illustre qu'il a d'ailleurs rencontré et qui n'est autre que saint Louis. Louis IX est en effet venu par trois fois au moins à saint Gilles. Les récits de Joinville (éd. 1874), de Mathieu Paris et les Itinera et mansiones recuillis par les Historiens de la France permettent de retracer un itinéraire qu'ont précisé ses biographes, Jean Richard (1983) et Jacques Le Goff (1996).

Quelques mois après sa mère la reine régente Blanche de Castille qui visite le tombeau de saint Gilles, le $1^{\text {er }}$ septembre 1247 (Bouisson, 1923-1930, p. 309), le roi prend la croix et se dirige à son tour vers la Provence. Le 12 juin 1248, il reçoit le bourdon et la besace du pèlerin à Saint-Denis; arrivé à Aigues-Mortes à la mi-août, il y embarque le 25 et quitte le port le 28 août. Parti de Paris, il s'arête à Corbeil, Melun, puis séjourne à Sens où se tient le chapitre provincial des Frères mineurs de France ; il passe en Bourgogne par Noyers-sur-Serein, Chablis (Apud Chableyas), puis par Vézelay et gagne Lyon où il rencontre le pape Innocent IV (Richard, 1983, p. 207 ; Le Goff, 1996, p. 537). Descendant le Rhône en bateau, il est arrêté au-dessus de Valence au château de La Roche de Glun (apud Rocham de Glui), dont le seigneur, Robert de Clérieu, exige des pèlerins un péage que le roi refuse de payer. Le souverain n'était d'ailleurs pas au bout de ses peines puisque la troupe est encore attaquée par des habitants à Avignon (Richard, 1983, p. 208; Le Goff, 1996, p. 185-187, 537)! Réfugié à Beaucaire (Belliquadrum), le roi poursuit vers Nîmes, Saint-Gilles et finalement Aigues-Mortes.

Six ans plus tard, au retour de la septième croisade, le roi suit un itinéraire un peu différent. Louis IX, embarqué à Acre le 25 avril 1254, débarque aux Salins de Hyères début juillet, entre le 3 et le 12 (Joinville, 1874, p. 358-359; Vernière, 1898, p. 18; Richard, 1983, p. 264 ; Le Goff, 1996, p. 210). Le roi s'arrête à Aix-en-Provence, visite la Sainte-Baume et rejoint Beaucaire où Joinville le quitte (Joinville, 1874, p. 364-365). Louis poursuit ensuite vers Saint-Gilles où il fait étape en juillet et rencontre l'abbé Guy Foulques, futur Clément IV (Le Goff, 1996, p. 221). En août, il poursuit sa route par Nîmes, Alès et Le Puy où il séjourne du 9 au 11 août. Il est ensuite à Brioude le 12, à Issoire le 13, il remonte par Clermont les 14 et 15, par Saint-Pourçain le 18, puis SaintBenoît-sur-Loire, Loury plutôt que Lorris (Apud Lorriacum), le château de Vincennes 
puis Saint-Denis ; il est de retour à Paris le 7 septembre (Richard, 1983, p. 265 ; Le Goff, 1996, p. 214, 537-538). Le choix de cet itinéraire répond à un double objectif : Jacques Le Goff souligne que le roi « remonte par l'Auvergne pour visiter les grands sanctuaires de pèlerinage du Puy et de Brioude", mais Antoine Vernière remarque que la multiplication des étapes aidait également Louis «à combler le déficit de ses finances en recueillant le droit de gîte dans toutes les villes ou monastères où il passait ».

En 1270 , le départ pour la huitième croisade conduit le roi à reprendre le chemin du Midi. Reproduisant le rituel de 1254, Louis IX se rend à Saint-Denis le 14 mars 1270 pour y recevoir le bourdon et la panetière (Richard, 1983, p. 556 ; Le Goff, 1996, p. 294). Le roi et ses trois fils quittent le Palais le lendemain, font étape à Vincennes, puis descendent par Villeneuve-Saint-Georges, Melun, Sens, Auxerre, Vézelay, Cluny, Mâcon, Lyon, Vienne et Beaucaire. Rejoint à Aigues-Mortes par Thibault de Navarre, le roi doit attendre la flotte génoise (Richard, 1983, p. 556). Les historiens de Saint-Gilles affirment que le roi est resté près de deux mois à attendre dans leur ville, mais les Itinera et mansiones le montrent plus mobile: il est à Sommières (apud Sumidrium) le 8 mai, séjourne à Nîmes du 13 au 23 mai au moins. Il va passer à Saint-Gilles la fête de la Pentecôte, le $1^{\text {er }}$ juin, et y reçoit les ambassadeurs de l'empereur grec Michel Paléologue (Charles-Roux, 1910, p. 228). Il reste les deux jours suivants au moins à Notre-Dame de Vauvert, puis rejoint Aigues-Mortes où il se trouve le 13 juin. Il donne ses dernières instructions à ses lieutenants le 25 juin, embarque le $1^{\mathrm{er}}$ juillet sur la nef La Montjoie qui appareille le 2.

Le choix de la voie rhodanienne caractérise aussi un autre croisé qui rejoint justement le roi à Saint-Gilles, le comte de Flandre Gui de Dampierre dont l'itinéraire est précisément documenté par les comptes de son clerc des finances, Jean Makiel (Rembry, 1881-1882, t. II, p. 587-590). Quittant son château de Male, près de Bruges, le 10 avril 1270, il arrive le 11 mai à Lyon, le 14 à Valence, le 20 mai à Beaucaire et le lendemain, veille de l'Ascension, il atteint Saint-Gilles où il retrouve le roi : Le mercredi vegile de l'Ascension, au mangier vint li cuens à S. Gile en Provence et fu la par II jors (Gaillard, 1856, p. 17, n. 37).

Dès le siècle précédent la voie fluviale a la faveur de certains pèlerins. Dans le Liber miraculorum sancti Egidii, un chevalier allemand nommé Guerritus, dénoncé comme voleur de bétail à Erfurt, échappe à la pendaison et vient rendre grâce à Saint-Gilles : «Accompagné de plusieurs pèlerins qui se hâtaient pour accomplir un semblable devoir de piété, il arriva à Vienne. Là, ils gagnèrent le port sur le Rhône et embarquèrent tous ensemble sur un navire de louage. » Réchappant cette fois d'un naufrage, le miraculé et ses compagnons atteignent finalement le sanctuaire (éd. Girault, 2007, n 12, p. 86-93). Dans la chanson de geste Aiol, le traître Makaire qui a fait prisonnier le héros et son épouse Mirabel, fuyant les troupes de Charlemagne lancées à sa poursuite, s'embarque avec ses captifs sur le Rhône qu'ils descendent jusqu'à Saint-Gilles. Ne prenant guère le temps de s'arrêter sur le tombeau du saint, ils poursuivent leur route par voie de terre vers le Somport et Pampelune : Ils sont venu al Rone, si trovent la navie. / [...] / Par le Rosne governent et a grant force vindrent : / Tout contreval s'en vont enfressi c'a Saint Gille, / Del cors saint n'ont que faire, il n'i tornerent mie(éd. Normand, Raynaud, v. 9555-9563, 9566). 


\section{La route d'Aurillac et la dômerie d'Aubrac}

Il est un autre itinéraire que Raymond Oursel n'a pas hésité à ajouter à la liste des "Chemins de Saint-Gilles ». Néanmoins il ne s'agit pas d'une appellation mentionnée par les sources médiévales, mais d'un itinéraire reconstitué par l'historien grâce à une méthode simple, puisque selon Oursel, suivi par Marcel Girault, il suffit, pour retrouver l'itinéraire que pouvaient suivre les pèlerins entre Aurillac, Nîmes et Saint-Gilles, de relier ces villes par une ligne droite, sur laquelle se situent les étapes de Laguiole, Aubrac, Chirac, Chanac; au-delà on suit l'ancien chemin de Marvejols à Nîmes qui existe encore par Ispagnac et Florac; puis on traverse le can de l'Hospitalet, Pompidou, la corniche des Cévennes, Saint-Jean-du-Gard et Anduze (Oursel, 1978, p. 21-35 ; Girault, 1990, p. 249-280). Le passage de chemins suivant les crêtes ou les vallées, utilisant les drailles de transhumance, traversant le causse de Sauveterre et le causse Méjean est attesté, mais leur emprunt par des pèlerins historiques n'est à ma connaissance pas précisément documenté (Oursel, 1967 ; Sors, 1967 ; Hamon, 1995).

Cependant, le point central de cet itinéraire est bien, selon le mot de Raymond Oursel, un « carrefour des chemins de pèlerinages " : il s'agit de l'hôpital ou dômerie d'Aubrac (Advielle, 1874 ; Debat, 1985 ; Péricard-Méa, 2000, p. 325-327 ; Petit, 2005). Un certain Alard ou Adalard, seigneur flamand, aurait fondé vers 1120 dans ce « lieu d'horreur et de vaste solitude » une communauté double vouée à l'accueil des étrangers et pèlerins. L'hôpital d'Aubrac est une étape bien connue sur la route du Puy, bien qu'elle ne soit pas citée par le Guide du pèlerin. Un vidimus de 1324 d'une bulle d'Honorius III de 1226 confirmant celle de son prédécesseur Innocent III développe le récit de la fondation et confirme le passage en ce lieu de nombreux pèlerins: « Alard, ce très heureux serviteur de Dieu, commença à y bâtir une église et un hôpital en l'honneur de Jésus Christ et de la glorieuse Marie toujours vierge pour y recevoir, ramasser et conforter les pauvres et les infirmes, les aveugles, les faibles, les boiteux, les sourds et muets et tous les pèlerins qui passaient sur ces montagnes pour aller visiter les églises de Sainte-Marie de Rocamadour, de Saint-Jacques, de Saint-Sauveur d'Oviedo, de Saint-Dominique d'Estremadoure et de nombreux autres sanctuaires, ainsi que ceux qui iront visiter le Sépulcre de Notre Seigneur» (éd . Rigal, Verlaguet, 1913, t. I, nº 17 p. 24). Dans une supplique adressée au roi de France au début du XIVesiècle, les doms d'Aubrac développent encore davantage cette liste des sanctuaires vers lesquels marchent les pèlerins accueillis à Aubrac; reprenant l'énumération précédente, ils y ajoutent les sanctuaires mariaux de Notre-Dame du Puy et de Quézac (en Lozère), de Saint-Antoine en Viennois et des apôtres à Rome (Girault, 1990, p. 250). Saint-Antoine désigne une route qui ne peut être que celle du Puy prolongée.

On remarque que Saint-Gilles n'y est pas cité. Pourtant des pèlerins de Saint-Gilles devaient bien fréquenter l'hôpital d'Aubrac : il semble en effet que la première église était dédiée à saint Gilles, et que c'est seulement plus tard qu'elle fut placée sous le vocable de Notre-Dame-des-Pauvres (Girault, 1990, p. 252). La dômerie d'Aubrac possédait un prieuré à Anduze quand l'abbaye de Saint-Gilles en détenait un à SaintJean-du-Gard (Girault, 1990, p. 275-276 ; Magnani, 2004) : ainsi l'accueil des pèlerins était-il contrôlé par des possessions croisées. Enfin, les pèlerins vers Rome et la Terre sainte qui s'embarquaient en Méditerranée devaient le faire à Saint-Gilles et à partir du XIII ${ }^{e}$ siècle à Aigues-Mortes. C'est pourquoi Aubrac apparaît bien comme une étape vraisemblable sinon un point de passage obligé pour les pèlerins de Saint-Gilles venant 
de l'Ouest, et donc un lieu de rencontre des pèlerins de Saint-Gilles et de Saint-Jacques à l'instar du Puy pour les pèlerins venus du Nord. Néanmoins, on ne peut que s'interroger sur les raisons de l'absence d'allusion au sanctuaire provençal dans les énumérations de pèlerins fournies par les documents de l'histoire d'Aubrac. Saint-Jacques apparaissent dans les itinéraires tant historiques que littéraires vers SaintGilles: sur la via Tolosana, nous avons retrouvé non seulement la cité de Saint-Gilles même, mais aussi Arles, Toulouse, Montpellier et Saint-Guilhem-le-Désert ; sur la Via podensis, nous avons vu l'importance du sanctuaire éponyme du Puy ; sur la troisième route, nous avons retrouvé Vézelay et Périgueux (ville d'origine du pèlerin blessé dans la crypte) ; et enfin, à l'origine de la route dite de Tours, nous avons rencontré Orléans, ville donnée comme point de départ dans le chapitre VIII... S'y ajoutent d'autres carrefours, omis dans le Guide, et parfois à l'écart des chemins qu'il décrit, comme Bussy, l'hôpital d'Aubrac ou l'étape plus incertaine de Brioude.

Comment caractériser ces points de jonctions? Il s'agit parfois de tronçons communs, comme entre Saint-Gilles et Toulouse, qui nous rappellent que les routes peuvent s'emprunter en deux sens; plus souvent il s'agit de nœuds routiers, où les itinéraires se croisent, nœuds caractérisés par la présence d'une infrastructure d'accueil (comme à Aubrac), ou d'un sanctuaire éminent lui-même but d'un grand pèlerinage (Toulouse, Saint-Guilhem, Le Puy...). Est-ce l'indice d'un réseau de lieux de pèlerinage qui se renverraient les pèlerins? L'hagiographie et l'épopée mettent en scène de nombreux pèlerins «collectionneurs » qui vont ainsi d'un sanctuaire à l'autre... Toutefois, qu'il s'agisse d'une stratégie délibérée et coordonnée paraît peu probable; une démarche concurrentielle me semble plus plausible. J'ai émis ailleurs l'hypothèse que les quatre routes décrites par le Guide partaient de grands sanctuaires français, ou les traversaient, pour capter ainsi les publics de ces sanctuaires prestigieux afin de les 
drainer vers la Galice (Girault, 2001, p. 376-377; Girault, 2004, p. 146-147). Probablement en va-t-il de même pour les Chemins de Saint-Gilles.

En effet, comme l'a remarqué Alphonse Dupront, « le recours pèlerin exige ; sinon il se détourne, cherchant un saint ou une puissance sacrale de plus grande vertu» (Dupront, 1972, p. 731c). Un pèlerin insatisfait dans l'objet de sa quête est si j'ose dire, un «client " idéal pour un sanctuaire rival. Cette rivalité a donné naissance à ce que Lucien Musset a appelé des "captures de pèlerinage » et qu'on pourrait également qualifier de détournements de pèlerins. On a vu par exemple que le Liber miraculorum sancti Egidii relate la mésaventure de la possédée Grada, qui n'ayant pu être guérie à Saint-Clet de Rome ni à Borgo San Donnino, est finalement conduite à Saint-Gilles où elle obtient la guérison. Dans la littérature aussi, c'est Guillaume, le héros du roman L'Escoufle, qui va en vain prier à Rome et à Compostelle, Dusqu'a S. Jake et dusqu'a Rome, afin de retrouver sa fiancée, et qui, n'ayant pas obtenu satisfaction, s'adresse finalement à saint Gilles, « au saint qui ne manque jamais d'entendre l'homme qui le prie de tout cœur »: Al saint qui ne faut nul homme, / Ki de cuer li prit k'il ne l'oie (v. 6480-6481).

Inversement, des recueils de miracles compilés dans d'autres sanctuaires signalent le cas de pèlerins qui n'étant pas exaucés à Saint-Gilles s'adressent à d'autres saints. Le livre des Miracles de Trèves rapporte qu'un pèlerin bavarois nommé Engelbert et souffrant de maladie pulmonaire, déçu de n'être pas guéri à Saint-Gilles, accepte l'invitation d'un marchand de Trèves qui le conduit auprès des reliques de l'apôtre Matthias nouvellement découvertes dans sa ville (1127), où il est bien sûr exaucé (Köster, 1983, p. 96-97 ; Girault, 2007, p. 300). De même, en 1145, à Pâques, une aveugle originaire de Wilton, en Angleterre, parcourt la France en essayant de trouver la guérison. «Après avoir imploré en vain saint Denis à Paris, elle est en route vers SaintGilles lorsqu'elle rencontre une troupe de pèlerins qui s'en reviennent de Reims où ils ont été guéris par saint Gibrien. Elle décide alors de changer d'itinéraire et, guidée par sa fille, se rend à Reims, où elle est guérie le 18 mai. " (Sigal, 1969, p. 1533-1534; id., 1985, p. 217).

Ainsi, les chemins de Saint-Gilles et leurs carrefours avec les chemins de Rome, de Compostelle, et de bien d'autres pèlerinages, dessinent-ils une géographie à la fois pragmatique, imaginaire, littéraire et sacrée : ils apparaissent non seulement comme des témoignages du maillage routier de l'espace, mais aussi comme des pôles d'échanges de sacralités et autant de passages ouverts vers de nouvelles quêtes spirituelles.

\section{BIBLIOGRAPHIE}

Advielle V., 1874, Notice sur l'hôpital d'Aubrac, Bruges, imp. de Daveluy.

Albe E., 1907, Les Miracles de Notre-Dame de Roc-Amadour au XIIe siècle, Paris, Champion, 347 p. 
Andrieu-Guitrancourt P., 1938, L'archevêque Eudes Rigaud et la vie de l'Église au XIII siècle, Paris, Librairie du "Recueil Sirey", XI-464 p.

Bautier R.-H., 1963, « Recherches sur les routes de l'Europe médiévale, II : Le grand axe routier est-ouest du Midi de la France, d'Avignon à Toulouse ", Bulletin philologique et historique jusqu'à 1610 (1961), p. 285-305.

Bautier R.-H., 1991, Sur l'histoire économique de la France médiévale : la route, le fleuve, la foire, Aldershot, X-347 p. (Collected studies series, 340).

Bautier R.-H., Labory G. (éd. et trad.), Vie de Robert le Pieux, Paris, CNRS, 165 p.

Bédier J., 1908-1912, Les légendes épiques. Recherches sur la formation des chansons de geste, Paris, Champion, 4 vol.

Bernard A. (éd.), 1853, Cartulaire de l'abbaye de Savigny, Paris, Impr. impériale (Coll. de documents inédits sur l'histoire de France), 2 vol.

Bonnin Th. (éd.), 1852, Regestrum visitationum archiespiscopi Rothomagensis. Journal des visites pastorales d'Eudes Rigaud, archevêque de Rouen, 1248-1269, Rouen, A. Le Brument, VII-860 p.

Borg S. J. (éd.), 1967, Aye d'Avignon, Genève, Droz, 378 p. (Textes littéraires français).

Bouisson E., 1923-1930, « Pèlerinage de la reine régente Blanche de Castille au tombeau de saint Gilles, le $1^{\mathrm{er}}$ septembre 1247 », Bulletin du Comité de l'art chrétien de Nîmes, t. XI, p. 309.

Brown S. M., O'Sullivan J. F. (éd.), 1964, The Register of Eudes Rigaud, New York, Columbia university press, XXXVI-779 $\mathrm{p}$.

Charles-Roux J., 1910, Souvenirs du passé. Saint-Gilles, sa légende, son abbaye, ses coutumes, Paris, A. Lemerre, $355 \mathrm{p}$.

Chassaing A. (éd.), 1869-1874, Chroniques d'Etienne Médicis, bourgeois du Puy, Le Puy-en-Velay, impr. de M.-P. Marchessou, 2 vol.

Chassaing A. (éd.), 1885, Mémoires d'Antoine Jacmon, bourgeois du Puy, Le Puy-en-Velay, Impr. de Marchessou fils, XIV-XII-308 p.

Chassaing A., 1907, Dictionnaire topographique du département de la Haute-Loire, Paris, E. Leroux, Impr. nationale, XLIII-393 p.

Cloetta W. (éd.), 1906-1913, Les deux rédactions en vers du Moniage Guillaume, Paris, Firmin-Didot, 2 vol.(Société des anciens textes français).

Darlington O. G., 1940, The Travels of Odo Rigaud, archbishop of Rouen, Philadelphia, Times and News publishing $\mathrm{C}^{\circ}, \mathrm{IX}-89 \mathrm{p}$.

Davis A. J., 2006, The Holy Bureaucrat: Eudes Rigaud and Religious Reform in Thirteenth-Century Normandy,Ithaca (N.Y.) : Cornell University Press, X-268 p.

Debat A., 1985, « Gilbert de Cantobre, évêque de Rodez (1339-1349) en conflit avec les religieux de Sainte-Eulalie-d'Olt et d'Aubrac », Revue du Rouergue, $39^{\mathrm{e}}$ année, $4^{\mathrm{e}}$ série, $\mathrm{n}^{\circ}$ 1, p. 15-36.

Dembowski P.F. (éd.), 1969, Ami et Amile, Paris, Champion, XVI-131 p. (Les classiques français du Moyen Âge, 97).

Dupront A., 1972, « Pèlerinages et lieux sacrés », dans Encyclopaedia Universalis, Paris, Encyclopaedia universalis France, vol. XXI, 729-734.

Gaillard V., 1856, Archives du conseil de Flandre, ou recueil de documents inédits relatifs à l'histoire politique, judiciaire, artistique et littéraire..., Gand, Busscher frères, $502 \mathrm{p}$. 
Gicquel B., 2003, La Légende de Compostelle : le livre de saint Jacques, Paris, Tallandier, 760 p.

Gilles-Guibert M., 1962, « Noms des routes et des chemins dans le Midi de la France au Moyen Âge », Bulletin philologique et historique jusqu'à 1610 (1960), p. 1-39.

Girault M. et P.-G. (dir.), 2007, Livre des miracles de saint Gilles (Liber miraculorum sancti Egidii) : La vie d'un sanctuaire de pèlerinage au XII ${ }^{e}$ siècle, Orléans, Paradigme, 367 p.

Girault M. et P.-G., 2001, Visages de pèlerins au Moyen Âge. Les Pèlerinages européens dans l'art et l'épopée, Saint-Léger-Vauban, Zodiaque, 388 p.

Girault M., 1980, Edition critique et commentée du Procès-verbal de «La visitation du Chemin appellé Regordane » effectuée par Louis de Froidour en 1668, thèse d'histoire-archéologie, Tours, Université François-Rabelais, 3 vol.

Girault M., 1982, «L'itinéraire du Charroi de Nîmes : Chemin de Saint-Gilles et chemin de Regordane ", dans La chanson de geste et le mythe carolingien : mélanges René Louis, Saint-Père-sousVézelay, Musée archéologique régional, t. II, p. 1105-1116.

Girault M., 1986, Le chemin de Regordane, ancienne route d'Alès au Puy, Nîmes, Lacour, 310 p.

Girault M., 1990, Les chemins de Saint-Gilles, itinéraires de pèlerinage, Nîmes, Lacour, 345 p.

Girault P.-G., 2003, « Compostelle dans les chansons de geste françaises, une approche chronologique », Saint Jacques et la France. Actes du colloque des 18 et 19 janvier 2001, (dir. Adeline Rucquoi), Paris, Cerf (Cerf-Histoire), p. 331-347.

Girault P.-G., 2005, « Saint-Gilles y su peregrinación en el siglo XII en el Codex Calixtinus », dans Caucci von Saucken P. (éd.), Visitandum est... Santos y cultos en el Codex Calixtinus, Actas del VII Congreso Internacional de estudios Jacobeos (Santiago de Compostela, 16-19 septembre 2004), Santiago, Xerencia de Promoción do Camino de Santiago, p.129-147.

Girault P.-G., 2012, « Pèlerins chevaliers à Saint-Gilles au XII ${ }^{\mathrm{e}}$ siècle d'après le Liber miraculorum sancti Egidii, entre miracles et épopée ", in Le pèlerinage de l'Antiquité à nos jours, dir. A. Vauchez (Actes du $130^{\circ}$ Congrès national des sociétés historiques et scientifiques, La Rochelle, 18-23 avril 2005), Paris, éd. du CTHS, p. 49-65 (version électronique sur CD-Rom et http://cths.fr).

Guizot F. (éd.), 1824, «Vie du roi Robert », dans Collection des mémoires relatifs à l'histoire de France, t. VI, Paris, J.-L.-J. Brière, p. 359-414.

HAMON E., 1995, « Les relations entre l'Aubrac et le Quercy à la fin du Moyen Âge : échanges économiques et mouvements de populations autour de la transhumance des troupeaux ", Procèsverbaux de la Société des lettres, sciences et arts de l'Aveyron (1994), p. 759-773.

Henry P. (éd.), 1935, Les Enfances Guillaume, Paris, Société des anciens textes français, XLIII-174 p.

Herbers K., Santos Noia, M., 1998, Liber sancti Jacobi. Codex Calixtinus, Santiago de Compostela, Xunta de Galicia, XXX-337 p.

Hippeau C. (éd.), 1874-1877, Chanson du Chevalier au Cygne et de Godefroid de Bouillon, Paris, A. Aubry, 2 vol. (Collection des poètes français du Moyen Âge).

Joinville J. de, 1874, Histoire de saint Louis, éd. Wailly N. de, Paris, Firmin-Didot, XXXIV-690 p.

Jounel P., 1992, «Les voyages des papes en France au Moyen Âge », Mélanges des sciences religieuses. Revue des facultés catholiques de Lille, t. 49, p. 5-32.

Jugnot G., 1982, « Les chemins de pèlerinage dans la France médiévale », Flaran 2 (1980), L'homme et la route en Europe occidentale au Moyen Âge et aux temps modernes, Auch, Comité départemental du tourisme du Gers, p. 57-83. 
Kay S., Kibler W. (éd. et trad.), 1996, Raoul de Cambrai, Paris, Librairie générale française, 540 p. (Lettres gothiques).

Köster K., 1983, Pilgerzeichen und Pilgermuscheln von mittelalterlichen Santiagostrassen : S.Léonard, Rocamadour, S. Gilles, Santiago de Compostela, Neumünster, K. Wachholtz, 175 p. (Ausgrabungen in Schleswig. Berichte und Studien, 2).

Lachet C. (éd. et trad.), 1999, Le Charroi de Nîmes, Paris, Gallimard, 242 p. (Folio Classiques).

Lauranson-Rosaz C., 1987, L'Auvergne et ses marges (Velay, Gévaudan) du Ve au XI' siècle : la fin du monde antique?, Le Puy-en-Velay, Les Cahiers de la Haute-Loire, 494 p. En ligne sur le site : www.droit.u-clermont1.fr.

Le Goff J., 1996, Saint Louis, Paris, Gallimard, 976 p.

Macaire P., 2000. Montpellier et Saint-Guilhem sur le chemin de Saint-Jacques, Notre-Dame-deLondres, Le Plein des Sens, 175 p.

Magnani E., 2004, « Réseaux monastiques et réseaux de pouvoir : Saint-Gilles-du-Gard, du Languedoc à la Hongrie, IX ${ }^{\mathrm{e}}$-début XIII ${ }^{\mathrm{e}}$ siècle », Provence historique, t. LIV, nº 215, p. 3-26.

Maurice Valla, 1964, «Chemin du Puy et de Saint-Jacques. La Via Podiensis en Forez », Bulletin de la Diana, t. XXVIII, nº 6, p. 176-195, 214-252.

Meyer P., Longnon A. (éd.), 1882, Raoul de Cambrai, Paris, Firmin-Didot, CIV-384 p. (Société des anciens textes français, 14).

Moulinet D., Bruno Maes B., Vincent C. (dir.), 2009, Jubilé et culte marial (Moyen Âge-époque contemporaine). Actes du colloque international, Puy-en-Velay, 8 juin-10 juin 2005, Saint-

Étienne, Publications de l'Université de Saint-Étienne, $460 \mathrm{p}$.

Normand J., Raynaud G. (éd.), 1877, Aiol, Paris, Firmin-Didot, LXVII-350 p. (Société des anciens textes français).

Oursel R., 1967, « Chemins de transhumance, chemins de pèlerinage », Archéologia, n 14, p. 71-77.

Oursel R., 1978, « Le grand hôpital d'Aubrac, une fondation flamande au carrefour des chemins de pèlerinages ", Annales de l'École des Hautes Études de Gand, t. IX, p. 21-35.

Pastoureau M., 2004, Histoire symbolique du Moyen Âge, Paris, Seuil, 436 p.

Péricard-Méa D., 2000, Compostelle et cultes de saint Jacques au Moyen Âge, Paris, PUF, 385 p.

Perrier J.-L. (éd.), 1931, Le Charroi de Nîmes, Paris, Champion, VIII-78 p. (Classiques français du Moyen Âge, 66).

Petit C., 2005, L'hôpital-monastère Notre-Dame des Pauvres d'Aubrac, [Rodez], impr. Groupe Burlat, $54 \mathrm{p}$.

Raynaud G. (éd.), 1879, Élie de Saint-Gilles, Paris, Firmin Didot, XLIII-203 p. (Société des anciens textes français).

Rembry E., 1881-1882, Saint Gilles, sa vie, ses reliques, son culte en Belgique et dans le nord de la France. Essai d'hagiographie, Bruges, E. Gailliard, 2 vol.

Renart J., 1974, L'Escoufle, éd. Sweetser F., Genève, Droz, XLII-411 p. (Textes littéraires français, 211).

Richard J., 1983, Saint Louis, roi d'une France féodale, soutien de la Terre sainte, Paris, Fayard, 638 p.

Rigal J.-L., Verlaguet P.-A., 1913-1917, Documents sur l'ancien hôpital d'Aubrac, Rodez, Impr. de Carrère, X-686 p. (Archives historiques du Rouergue, 4). 
Sigal P.-A., 1969, « Maladie, pèlerinage et guérison au XII ${ }^{\mathrm{e}}$ siècle : les miracles de saint Gibrien à Reims ", Annales ESC, $24^{\mathrm{e}}$ année, $\mathrm{n}^{\circ}$ 6, p. 1533-1534.

Sigal P.-A., 1985, L'homme et le miracle dans la France médiévale, XI ${ }^{e}$-XII siècles, Paris, cerf, 349 p.

Sigal P.-A., 1993, « Saint-Gilles centre de pèlerinage au XIIe siècle : le témoignage des Miracula sancti Aegidii ", dans Hommage à R. Saint-Jean, Mémoires de la société archéologique de Montpellier, $\mathrm{t}$. XXI, p. 247-255.

Sors A., 1967, « Chemins de pèlerinage du Rouergue et du Quercy », Revue de Rouergue, $\mathrm{n}^{\circ}$ 21, p. 65-69.

Terracher A.-L. (éd.), 1909, La Chevalerie Vivien, Paris, Champion, XII-287 p.

Theis L., 1999, Robert le Pieux : le roi de l'an mil, Paris, Perrin, 268 p.

Vernière A., 1898, Itinéraires des rois de France et des papes dans l'Auvergne et le Velay, ClermontFerrand, impr. de Malleval, $27 \mathrm{p}$.

Vielliard J., 1950, Le Guide du pèlerin de Saint-Jacques de Compostelle, texte latin du XII ${ }^{e}$ siècle, $2^{\mathrm{e}}$ éd., Mâcon, impr. Protat frères, XIX-153 p.

Wilmotte M. (éd.), 1927, Guillaume d'Angleterre, Paris, Champion, XIV-133 p. (Classiques français du Moyen Âge, 55).

\section{RÉSUMÉS}

Souvent regardé comme une simple étape vers Compostelle, le sanctuaire de Saint-Gilles (Gard) était au Moyen Âge le but d'un pèlerinage important. Cet article explore les itinéraires qui y conduisaient, à travers le fameux Guide du pèlerin de Saint-Jacques, qu'il confronte aux autres sources écrites médiévales, notamment littéraires. Toulouse, Montpellier, Le Puy apparaissent comme des points de départ tant vers Saint-Gilles que vers Compostelle. Toutefois l'appellation Chemin de Saint-Gilles paraît avoir désigné l'itinéraire qui y conduisait depuis Orléans, documenté par le pèlerinage de Robert le Pieux, la chanson de geste du Charroi de Nîmes, les itinéraires de l'archevêque de Rouen Eudes Rigaud et du roi Saint Louis. Ces chemins empruntés vers l'un ou l'autre sanctuaire dessinent une autre géographie sacrée de la France médiévale.

Often considered as a simple step towards Compostela, the sanctuary of Saint-Gilles (Gard) was in the Middle Ages the goal of an important pilgrimage. This article explores the routes leading there, through the famous Pilgrim's Guide to Santiago, compared with other medieval written sources, especially literary texts. Toulouse, Montpellier, Le Puy appear as points of departure both towards Saint-Gilles and towards Compostela. Nevertheless the name Chemin de SaintGilles seems to have designated the route which led there from Orleans, documented by the pilgrimage of Robert le Pieux, the epic poem of the Charroi de Nîmes, the routes of the archbishop of Rouen Odo Rigaud and of the king Saint Louis. These paths taken towards one or the other sanctuary draw another sacred geography of medieval France.

\section{INDEX}

Mots-clés : Pèlerinage, Saint-Gilles (Chemins de), Chanson de geste, Guide du pèlerin de SaintJacques

Keywords : Pilgrimage, Saint-Gilles (Pilgrimage Roads to), Chanson de geste, Pilgrim's Guide to Santiago 
AUTEUR

PIERRE-GILLES GIRAULT

Conservateur en chef du patrimoine, administrateur du Monastère royal de Brou 